\title{
Approximating fixed points of multivalued $\rho$-nonexpansive mappings in modular function spaces
}

\author{
Safeer Hussain Khan ${ }^{1 *}$ and Mujahid Abbas $^{2}$ \\ Dedicated to Professor Wataru Takahashi on his 70th birthday.
}

*Correspondence:

safeer@qu.edu.qa;

safeerhussain5@yahoo.com

'Department of Mathematics,

Statistics and Physics, Qatar

University, Doha, 2713, Qatar

Full list of author information is

available at the end of the article

\begin{abstract}
The existence of fixed points of single-valued mappings in modular function spaces has been studied by many authors. The approximation of fixed points in such spaces via convergence of an iterative process for single-valued mappings has also been attempted very recently by Dehaish and Kozlowski (Fixed Point Theory Appl. 2012:118, 2012). In this paper, we initiate the study of approximating fixed points by the convergence of a Mann iterative process applied on multivalued $\rho$-nonexpansive mappings in modular function spaces. Our results also generalize the corresponding results of (Dehaish and Kozlowski in Fixed Point Theory Appl. 2012:118, 2012) to the case of multivalued mappings.
\end{abstract}

MSC: 47H09; 47H10; 54C60

Keywords: fixed point; multivalued $\rho$-nonexpansive mapping; iterative process; modular function space

\section{Introduction and preliminaries}

The theory of modular spaces was initiated by Nakano [1] in connection with the theory of ordered spaces, which was further generalized by Musielak and Orlicz [2]. The fixed point theory for nonlinear mappings is an important subject of nonlinear functional analysis and is widely applied to nonlinear integral equations and differential equations. The study of this theory in the context of modular function spaces was initiated by Khamsi $e t$ al. [3] (see also [4-8]). Kumam [9] obtained some fixed point theorems for nonexpansive mappings in arbitrary modular spaces. Kozlowski [10] has contributed a lot towards the study of modular function spaces both on his own and with his collaborators. Of course, most of the work done on fixed points in these spaces was of existential nature. No results were obtained for the approximation of fixed points in modular function spaces until recently Dehaish and Kozlowski [11] tried to fill this gap using a Mann iterative process for asymptotically pointwise nonexpansive mappings.

All above work has been done for single-valued mappings. On the other hand, the study of fixed points for multivalued contractions and nonexpansive mappings using the Hausdorff metric was initiated by Markin [12] (see also [13]). Later, an interesting and rich fixed point theory for such maps was developed which has applications in control theory, convex optimization, differential inclusion, and economics (see [14] and references cited

○2014 Khan and Abbas; licensee Springer. This is an Open Access article distributed under the terms of the Creative Commons Attribution License (http://creativecommons.org/licenses/by/2.0), which permits unrestricted use, distribution, and reproduction in any medium, provided the original work is properly cited. 
therein). Moreover, the existence of fixed points for multivalued nonexpansive mappings in uniformly convex Banach spaces was proved by Lim [15]. The theory of multivalued nonexpansive mappings is harder than the corresponding theory of single-valued nonexpansive mappings. Different iterative processes have been used to approximate the fixed points of multivalued nonexpansive mappings in Banach spaces.

Dhompongsa et al. [5] have proved that every $\rho$-contraction $T: C \rightarrow F_{\rho}(C)$ has a fixed point where $\rho$ is a convex function modular satisfying the so-called $\Delta_{2}$-type condition, $C$ is a nonempty $\rho$-bounded $\rho$-closed subset of $L_{\rho}$ and $F_{\rho}(C)$ a family of $\rho$-closed subsets of $C$. By using this result, they asserted the existence of fixed points for multivalued $\rho$-nonexpansive mappings. Again their results are existential in nature. See also Kutbi and Latif [16].

In this paper, we approximate fixed points of $\rho$-nonexpansive multivalued mappings in modular function spaces using a Mann iterative process. We make the first ever effort to fill the gap between the existence and the approximation of fixed points of $\rho$-nonexpansive multivalued mappings in modular function spaces. In a way, the corresponding results of Dehaish and Kozlowski [11] are also generalized to the case of multivalued mappings.

Some basic facts and notation needed in this paper are recalled as follows.

Let $\Omega$ be a nonempty set and $\Sigma$ a nontrivial $\sigma$-algebra of subsets of $\Omega$. Let $\mathcal{P}$ be a $\delta$-ring of subsets of $\Omega$, such that $E \cap A \in \mathcal{P}$ for any $E \in \mathcal{P}$ and $A \in \Sigma$. Let us assume that there exists an increasing sequence of sets $K_{n} \in \mathcal{P}$ such that $\Omega=\bigcup K_{n}$ (for instance, $\mathcal{P}$ can be the class of sets of finite measure in a $\sigma$-finite measure space). By $1_{A}$, we denote the characteristic function of the set $A$ in $\Omega$. By $\mathcal{E}$ we denote the linear space of all simple functions with supports from $\mathcal{P}$. By $\mathcal{M}_{\infty}$ we will denote the space of all extended measurable functions, i.e., all functions $f: \Omega \rightarrow[-\infty, \infty]$ such that there exists a sequence $\left\{g_{n}\right\} \subset \mathcal{E},\left|g_{n}\right| \leq|f|$ and $g_{n}(\omega) \rightarrow f(\omega)$ for all $\omega \in \Omega$.

Definition 1 Let $\rho: \mathcal{M}_{\infty} \rightarrow[0, \infty]$ be a nontrivial, convex and even function. We say that $\rho$ is a regular convex function pseudomodular if

(1) $\rho(0)=0$;

(2) $\rho$ is monotone, i.e., $|f(\omega)| \leq|g(\omega)|$ for any $\omega \in \Omega$ implies $\rho(f) \leq \rho(g)$, where $f, g \in \mathcal{M}_{\infty}$;

(3) $\rho$ is orthogonally subadditive, i.e., $\rho\left(f 1_{A \cup B}\right) \leq \rho\left(f 1_{A}\right)+\rho\left(f 1_{B}\right)$ for any $A, B \in \Sigma$ such that $A \cap B \neq \phi, f \in \mathcal{M}_{\infty}$;

(4) $\rho$ has Fatou property, i.e., $\left|f_{n}(\omega)\right| \uparrow|f(\omega)|$ for all $\omega \in \Omega$ implies $\rho\left(f_{n}\right) \uparrow \rho(f)$, where $f \in \mathcal{M}_{\infty}$;

(5) $\rho$ is order continuous in $\mathcal{E}$, i.e., $g_{n} \in \mathcal{E}$, and $\left|g_{n}(\omega)\right| \downarrow 0$ implies $\rho\left(g_{n}\right) \downarrow 0$.

A set $A \in \Sigma$ is said to be $\rho$-null if $\rho\left(g 1_{A}\right)=0$ for every $g \in \mathcal{E}$. A property $p(\omega)$ is said to hold $\rho$-almost everywhere ( $\rho$-a.e.) if the set $\{\omega \in \Omega: p(\omega)$ does not hold $\}$ is $\rho$-null. As usual, we identify any pair of measurable sets whose symmetric difference is $\rho$-null as well as any pair of measurable functions differing only on a $\rho$-null set. With this in mind we define

$$
\mathcal{M}(\Omega, \Sigma, \mathcal{P}, \rho)=\left\{f \in \mathcal{M}_{\infty}:|f(\omega)|<\infty \rho \text {-a.e. }\right\}
$$

where $f \in \mathcal{M}(\Omega, \Sigma, \mathcal{P}, \rho)$ is actually an equivalence class of functions equal $\rho$-a.e. rather than an individual function. Where no confusion exists we will write $\mathcal{M}$ instead of $\mathcal{M}(\Omega, \Sigma, \mathcal{P}, \rho)$. 
Definition 2 Let $\rho$ be a regular function pseudomodular. We say that $\rho$ is a regular convex function modular if $\rho(f)=0$ implies $f=0 \rho$-a.e.

It is known (see [10]) that $\rho$ satisfies the following properties:

(1) $\rho(0)=0$ iff $f=0 \rho$-a.e.

(2) $\rho(\alpha f)=\rho(f)$ for every scalar $\alpha$ with $|\alpha|=1$ and $f \in \mathcal{M}$.

(3) $\rho(\alpha f+\beta g) \leq \rho(f)+\rho(g)$ if $\alpha+\beta=1, \alpha, \beta \geq 0$ and $f, g \in \mathcal{M}$.

$\rho$ is called a convex modular if, in addition, the following property is satisfied:

(3') $\rho(\alpha f+\beta g) \leq \alpha \rho(f)+\beta \rho(g)$ if $\alpha+\beta=1, \alpha, \beta \geq 0$ and $f, g \in \mathcal{M}$.

Definition 3 The convex function modular $\rho$ defines the modular function space $L_{\rho}$ as

$$
L_{\rho}=\{f \in \mathcal{M} ; \rho(\lambda f) \rightarrow 0 \text { as } \lambda \rightarrow 0\} .
$$

Generally, the modular $\rho$ is not subadditive and therefore does not behave as a norm or a distance. However, the modular space $L_{\rho}$ can be equipped with an $F$-norm defined by

$$
\|f\|_{\rho}=\inf \left\{\alpha>0: \rho\left(\frac{f}{\alpha}\right) \leq \alpha\right\}
$$

In the case $\rho$ is convex modular,

$$
\|f\|_{\rho}=\inf \left\{\alpha>0: \rho\left(\frac{f}{\alpha}\right) \leq 1\right\}
$$

defines a norm on the modular space $L_{\rho}$, and it is called the Luxemburg norm.

The following uniform convexity type properties of $\rho$ can be found in [11].

Definition 4 Let $\rho$ be a nonzero regular convex function modular defined on $\Omega$. Let $t \in$ $(0,1), r>0, \varepsilon>0$. Define

$$
D\left(r_{1}, \varepsilon\right)=\left\{(f, g): f, g \in L_{\rho}, \rho(f) \leq r, \rho(g) \leq r, \rho(f-g) \geq \varepsilon r\right\} .
$$

Let

$$
\delta_{1}^{t}(r, \varepsilon)=\inf \left\{1-\frac{1}{r} \rho(t f+(1-t) g):(f, g) \in D\left(r_{1}, \varepsilon\right)\right\} \quad \text { if } D\left(r_{1}, \varepsilon\right) \neq \phi,
$$

and $\delta_{1}(r, \varepsilon)=1$ if $D\left(r_{1}, \varepsilon\right)=\phi$.

As a conventional notation, $\delta_{1}=\delta_{1}^{\frac{1}{2}}$.

Definition 5 A nonzero regular convex function modular $\rho$ is said to satisfy (UC1) if for every $r>0, \varepsilon>0, \delta_{1}(r, \varepsilon)>0$. Note that for every $r>0, D_{1}(r, \varepsilon) \neq \phi$ for $\varepsilon>0$ small enough. $\rho$ is said to satisfy (UUC1) if for every $s \geq 0, \varepsilon>0$, there exists $\eta_{1}(s, \varepsilon)>0$ depending only upon $s$ and $\varepsilon$ such that $\delta_{1}(r, \varepsilon)>\eta_{1}(s, \varepsilon)>0$ for any $r>s$.

Definition 6 Let $L_{\rho}$ be a modular space. The sequence $\left\{f_{n}\right\} \subset L_{\rho}$ is called: 
- $\rho$-convergent to $f \in L_{\rho}$ if $\rho\left(f_{n}-f\right) \rightarrow 0$ as $n \rightarrow \infty$;

- $\rho$-Cauchy, if $\rho\left(f_{n}-f_{m}\right) \rightarrow 0$ as $n$ and $m \rightarrow \infty$.

Consistent with [17], the $\rho$-distance from an $f \in L_{\rho}$ to a set $D \subset L_{\rho}$ is given as follows:

$$
\operatorname{dist}_{\rho}(f, D)=\inf \{\rho(f-h): h \in D\}
$$

Definition 7 A subset $D \subset L_{\rho}$ is called:

- $\rho$-closed if the $\rho$-limit of a $\rho$-convergent sequence of $D$ always belongs to $D$;

- $\rho$-a.e. closed if the $\rho$-a.e. limit of a $\rho$-a.e. convergent sequence of $D$ always belongs to $D$;

- $\rho$-compact if every sequence in $D$ has a $\rho$-convergent subsequence in $D$;

- $\rho$-a.e. compact if every sequence in $D$ has a $\rho$-a.e. convergent subsequence in $D$;

- $\rho$-bounded if

$$
\operatorname{diam}_{\rho}(D)=\sup \{\rho(f-g): f, g \in D\}<\infty
$$

A set $D \subset L_{\rho}$ is called $\rho$-proximinal if for each $f \in L_{\rho}$ there exists an element $g \in D$ such that $\rho(f-g)=\operatorname{dist}_{\rho}(f, D)$. We shall denote the family of nonempty $\rho$-bounded $\rho$-proximinal subsets of $D$ by $P_{\rho}(D)$, the family of nonempty $\rho$-closed $\rho$-bounded subsets of $D$ by $C_{\rho}(D)$ and the family of $\rho$-compact subsets of $D$ by $K_{\rho}(D)$. Let $H_{\rho}(\cdot, \cdot)$ be the $\rho$-Hausdorff distance on $C_{\rho}\left(L_{\rho}\right)$, that is,

$$
H_{\rho}(A, B)=\max \left\{\sup _{f \in A} \operatorname{dist}_{\rho}(f, B), \sup _{g \in B} \operatorname{dist}_{\rho}(g, A)\right\}, \quad A, B \in C_{\rho}\left(L_{\rho}\right) .
$$

A multivalued mapping $T: D \rightarrow C_{\rho}\left(L_{\rho}\right)$ is said to be $\rho$-nonexpansive if

$$
H_{\rho}(T f, T g) \leq \rho(f-g), \quad f, g \in D .
$$

A sequence $\left\{t_{n}\right\} \subset(0,1)$ is called bounded away from 0 if there exists $a>0$ such that $t_{n} \geq a$ for every $n \in \mathbb{N}$. Similarly, $\left\{t_{n}\right\} \subset(0,1)$ is called bounded away from 1 if there exists $b<1$ such that $t_{n} \leq b$ for every $n \in \mathbb{N}$.

Lemma 1 (Lemma 3.2 [11]) Let $\rho$ satisfy $(U U C 1)$ and let $\left\{t_{k}\right\} \subset(0,1)$ be bounded away from 0 and 1 . If there exists $R>0$ such that

$$
\limsup _{n \rightarrow \infty} \rho\left(f_{n}\right) \leq R, \quad \limsup _{n \rightarrow \infty} \rho\left(g_{n}\right) \leq R
$$

and

$$
\lim _{n \rightarrow \infty} \rho\left(t_{n} f_{n}+\left(1-t_{n}\right) g_{n}\right)=R,
$$

then $\lim _{n \rightarrow \infty} \rho\left(f_{n}-g_{n}\right)=0$.

The above lemma is an analogue of a famous lemma due to Schu [18] in Banach spaces. A function $f \in L_{\rho}$ is called a fixed point of $T: L_{\rho} \rightarrow P_{\rho}(D)$ if $f \in T f$. The set of all fixed points of $T$ will be denoted by $F_{\rho}(T)$. 
Lemma 2 Let $T: D \rightarrow P_{\rho}(D)$ be a multivalued mapping and

$$
P_{\rho}^{T}(f)=\left\{g \in T f: \rho(f-g)=\operatorname{dist}_{\rho}(f, T f)\right\} .
$$

Then the following are equivalent:

(1) $f \in F_{\rho}(T)$, that is, $f \in T f$.

(2) $P_{\rho}^{T}(f)=\{f\}$, that is, $f=g$ for each $g \in P_{\rho}^{T}(f)$.

(3) $f \in F\left(P_{\rho}^{T}(f)\right)$, that is, $f \in P_{\rho}^{T}(f)$. Further $F_{\rho}(T)=F\left(P_{\rho}^{T}(f)\right)$ where $F\left(P_{\rho}^{T}(f)\right)$ denotes the set of fixed points of $P_{\rho}^{T}(f)$.

Proof (1) $\Longrightarrow(2)$. Since $f \in F_{\rho}(T) \Longrightarrow f \in T f$, so $\operatorname{dist}_{\rho}(f, T f)=0$. Therefore, for any $g \in$ $P_{\rho}^{T}(f), \rho(f-g)=\operatorname{dist}_{\rho}(f, T f)=0$ implies that $\rho(f-g)=0$. Hence $f=g$. That is, $P_{\rho}^{T}(f)=\{f\}$.

(2) $\Longrightarrow(3)$. Obvious.

(3) $\Longrightarrow(1)$. Since $f \in F\left(P_{\rho}^{T}(f)\right)$, so by definition of $P_{\rho}^{T}(f)$ we have $\operatorname{dist}_{\rho}(f, T f)=\rho(f-f)=0$. Thus $f \in T f$ by $\rho$-closedness of $T f$.

Definition 8 A multivalued mapping $T: D \rightarrow C_{\rho}(D)$ is said to satisfy condition (I) if there exists a nondecreasing function $l:[0, \infty) \rightarrow[0, \infty)$ with $l(0)=0, l(r)>0$ for all $r \in(0, \infty)$ such that $\operatorname{dist}_{\rho}(f, T f) \geq l\left(\operatorname{dist}_{\rho}\left(f, F_{\rho}(T)\right)\right)$ for all $f \in D$.

It is a multivalued version of condition (I) of Senter and Dotson [19] in the framework of modular function spaces.

\section{Main results}

We prove a key result giving a major support to our $\rho$-convergence result for approximating fixed points of multivalued $\rho$-nonexpansive mappings in modular function spaces using a Mann iterative process.

Theorem 1 Let $\rho$ satisfy (UUC1) and D a nonempty $\rho$-closed, $\rho$-bounded and convex subset of $L_{\rho}$. Let $T: D \rightarrow P_{\rho}(D)$ be a multivalued mapping such that $P_{\rho}^{T}$ is a $\rho$-nonexpansive mapping. Suppose that $F_{\rho}(T) \neq \phi$. Let $\left\{f_{n}\right\} \subset D$ be defined by the Mann iterative process:

$$
f_{n+1}=\left(1-\alpha_{n}\right) f_{n}+\alpha_{n} u_{n}
$$

where $u_{n} \in P_{\rho}^{T}\left(f_{n}\right)$ and $\left\{\alpha_{n}\right\} \subset(0,1)$ is bounded away from both 0 and 1 . Then

$$
\lim _{n \rightarrow \infty} \rho\left(f_{n}-c\right) \text { exists for all } c \in F_{\rho}(T)
$$

and

$$
\lim _{n \rightarrow \infty} \rho\left(f_{n}-P_{\rho}^{T}\left(f_{n}\right)\right)=0 .
$$

Proof Let $c \in F_{\rho}(T)$. By Lemma 2, $P_{\rho}^{T}(c)=\{c\}$. Moreover, by the same lemma, $F_{\rho}(T)=$ $F\left(P_{\rho}^{T}\right)$. To prove that $\lim _{n \rightarrow \infty} \rho\left(f_{n}-c\right)$ exists for all $c \in F_{\rho}(T)$, consider

$$
\begin{aligned}
\rho\left(f_{n+1}-c\right) & =\rho\left[\left(1-\alpha_{n}\right) f_{n}+\alpha_{n} u_{n}-c\right] \\
& =\rho\left[\left(1-\alpha_{n}\right)\left(f_{n}-c\right)+\alpha_{n}\left(u_{n}-c\right)\right] .
\end{aligned}
$$


By convexity of $\rho$, we have

$$
\begin{aligned}
\rho\left(f_{n+1}-c\right) & \leq\left(1-\alpha_{n}\right) \rho\left(f_{n}-c\right)+\alpha_{n} \rho\left(u_{n}-c\right) \\
& \leq\left(1-\alpha_{n}\right) H_{\rho}\left(P_{\rho}^{T}\left(f_{n}\right), P_{\rho}^{T}(c)\right)+\alpha_{n} H_{\rho}\left(P_{\rho}^{T}\left(f_{n}\right), P_{\rho}^{T}(c)\right) \\
& \leq\left(1-\alpha_{n}\right) \rho\left(f_{n}-c\right)+\alpha_{n} \rho\left(f_{n}-c\right) \\
& =\rho\left(f_{n}-c\right) .
\end{aligned}
$$

Hence $\lim _{n \rightarrow \infty} \rho\left(f_{n}-c\right)$ exists for each $c \in F_{\rho}(T)$.

Suppose that

$$
\lim _{n \rightarrow \infty} \rho\left(f_{n}-c\right)=L
$$

where $L \geq 0$.

We now prove that

$$
\lim _{n \rightarrow \infty} \rho\left(f_{n}-P_{\rho}^{T}\left(f_{n}\right)\right)=0 .
$$

As $\operatorname{dist}_{\rho}\left(f_{n}, P_{\rho}^{T}\left(f_{n}\right)\right) \leq \rho\left(f_{n}-u_{n}\right)$, it suffices to prove that

$$
\lim _{n \rightarrow \infty} \rho\left(f_{n}-u_{n}\right)=0 .
$$

Since

$$
\rho\left(u_{n}-c\right) \leq H_{\rho}\left(P_{\rho}^{T}\left(f_{n}\right), P_{\rho}^{T}(c)\right) \leq \rho\left(f_{n}-c\right),
$$

therefore

$$
\limsup _{n \rightarrow \infty} \rho\left(u_{n}-c\right) \leq \limsup _{n \rightarrow \infty} \rho\left(f_{n}-c\right)
$$

and so in view of (2.1), we have

$$
\limsup _{n \rightarrow \infty} \rho\left(u_{n}-c\right) \leq L
$$

As

$$
\begin{aligned}
\lim _{n \rightarrow \infty} \rho\left(f_{n+1}-c\right) & =\lim _{n \rightarrow \infty} \rho\left[\left(1-\alpha_{n}\right) f_{n}+\alpha_{n} u_{n}-c\right] \\
& =\lim _{n \rightarrow \infty} \rho\left[\left(1-\alpha_{n}\right)\left(f_{n}-c\right)+\alpha_{n}\left(u_{n}-c\right)\right] \\
& =L,
\end{aligned}
$$

from (2.1), (2.2), (2.3), and Lemma 1, we have

$$
\lim _{n \rightarrow \infty} \rho\left(f_{n}-u_{n}\right)=0
$$

Hence

$$
\lim _{n \rightarrow \infty} \operatorname{dist}_{\rho}\left(f_{n}, P_{\rho}^{T}\left(f_{n}\right)\right)=0 .
$$


Now we are all set for our convergence result for approximating fixed points of multivalued $\rho$-nonexpansive mappings in modular function spaces using the Mann iterative process as follows.

Theorem 2 Let $\rho$ satisfy (UUC1) and D a nonempty $\rho$-compact, $\rho$-bounded and convex subset of $L_{\rho}$. Let $T: D \rightarrow P_{\rho}(D)$ be a multivalued mapping such that $P_{\rho}^{T}$ is $\rho$-nonexpansive mapping. Suppose that $F_{\rho}(T) \neq \phi$. Let $\left\{f_{n}\right\}$ be as defined in Theorem 1 . Then $\left\{f_{n}\right\} \rho$-converges to a fixed point of $T$.

Proof From $\rho$-compactness of $D$, there exists a subsequence $\left\{f_{n_{k}}\right\}$ of $\left\{f_{n}\right\}$ such that $\lim _{k \rightarrow \infty}\left(f_{n_{k}}-q\right)=0$ for some $q \in D$. To prove that $q$ is a fixed point of $T$, let $g$ be an arbitrary point in $P_{\rho}^{T}(q)$ and $f$ in $P_{\rho}^{T}\left(f_{n_{k}}\right)$. Note that

$$
\begin{aligned}
\rho\left(\frac{q-g}{3}\right) & =\rho\left(\frac{q-f_{n_{k}}}{3}+\frac{f_{n_{k}}-f}{3}+\frac{f-g}{3}\right) \\
& \leq \frac{1}{3} \rho\left(q-f_{n_{k}}\right)+\frac{1}{3} \rho\left(f_{n_{k}}-f\right)+\frac{1}{3} \rho(f-g) \\
& \leq \rho\left(q-f_{n_{k}}\right)+\operatorname{dist}_{\rho}\left(f_{n_{k}}, P_{\rho}^{T}\left(f_{n_{k}}\right)\right)+\operatorname{dist}_{\rho}\left(P_{\rho}^{T}\left(f_{n_{k}}\right), g\right) \\
& \leq \rho\left(q-f_{n_{k}}\right)+\operatorname{dist}_{\rho}\left(f_{n_{k}}, P_{\rho}^{T}\left(f_{n_{k}}\right)\right)+H_{\rho}\left(P_{\rho}^{T}\left(f_{n_{k}}\right), P_{\rho}^{T}(q)\right) \\
& \leq \rho\left(q-f_{n_{k}}\right)+\operatorname{dist}_{\rho}\left(f_{n_{k}}, P_{\rho}^{T}\left(f_{n_{k}}\right)\right)+\rho\left(q-f_{n_{k}}\right) .
\end{aligned}
$$

By Theorem 1, we have $\lim _{n \rightarrow \infty} \operatorname{dist}_{\rho}\left(f_{n}, P_{\rho}^{T}\left(f_{n}\right)\right)=0$. This gives $\rho\left(\frac{q-g}{3}\right)=0$. Hence $q$ is a fixed point of $P_{\rho}^{T}$. Since the set of fixed points of $P_{\rho}^{T}$ is the same as that of $T$ by Lemma 2, $\left\{f_{n}\right\} \rho$-converges to a fixed point of $T$.

Theorem 3 Let $\rho$ satisfy (UUC1) and D a nonempty $\rho$-closed, $\rho$-bounded and convex subset of $L_{\rho}$. Let $T: D \rightarrow P_{\rho}(D)$ be a multivalued mapping with and $F_{\rho}(T) \neq \phi$ and satisfying condition (I) such that $P_{\rho}^{T}$ is $\rho$-nonexpansive mapping. Let $\left\{f_{n}\right\}$ be as defined in Theorem 1 . Then $\left\{f_{n}\right\}$-converges to a fixed point of $T$.

Proof From Theorem 1, $\lim _{n \rightarrow \infty} \rho\left(f_{n}-c\right)$ exists for all $c \in F\left(P_{\rho}^{T}\right)=F_{\rho}(T)$. If $\lim _{n \rightarrow \infty} \rho\left(f_{n}-\right.$ $c)=0$, there is nothing to prove. We assume $\lim _{n \rightarrow \infty} \rho\left(f_{n}-c\right)=L>0$. Again from Theorem $1, \rho\left(f_{n+1}-c\right) \leq \rho\left(f_{n}-c\right)$ so that

$$
\operatorname{dist}_{\rho}\left(f_{n+1}, F_{\rho}(T)\right) \leq \operatorname{dist}_{\rho}\left(f_{n}, F_{\rho}(T)\right) .
$$

Hence $\lim _{n \rightarrow \infty} \operatorname{dist}_{\rho}\left(f_{n}, F_{\rho}(T)\right)$ exists. We now prove that $\lim _{n \rightarrow \infty} \operatorname{dist}_{\rho}\left(f_{n}, F_{\rho}(T)\right)=0$. By using condition (I) and Theorem 1 , we have

$$
\lim _{n \rightarrow \infty} l\left(\operatorname{dist}_{\rho}\left(f_{n}, F_{\rho}(T)\right)\right) \leq \lim _{n \rightarrow \infty} \operatorname{dist}_{\rho}\left(f_{n}, T f_{n}\right)=0 .
$$

That is,

$$
\lim _{n \rightarrow \infty} l\left(\operatorname{dist}_{\rho}\left(f_{n}, F_{\rho}(T)\right)\right)=0 .
$$

Since $l$ is a nondecreasing function and $l(0)=0$, it follows that $\lim _{n \rightarrow \infty} \operatorname{dist}_{\rho}\left(f_{n}, F_{\rho}(T)\right)=0$. 
Next, we show that $\left\{f_{n}\right\}$ is a $\rho$-Cauchy sequence in $D$. Let $\varepsilon>0$ be arbitrarily chosen. Since $\lim _{n \rightarrow \infty} \operatorname{dist}_{\rho}\left(f_{n}, F_{\rho}(T)\right)=0$, there exists a constant $n_{0}$ such that for all $n \geq n_{0}$, we have

$$
\operatorname{dist}_{\rho}\left(f_{n}, F_{\rho}(T)\right)<\frac{\varepsilon}{2}
$$

In particular, $\inf \left\{\rho\left(f_{n_{0}}-c\right): c \in F_{\rho}(T)\right\}<\frac{\varepsilon}{2}$. There must exist a $c^{*} \in F_{\rho}(T)$ such that

$$
\rho\left(f_{n_{0}}-c^{*}\right)<\varepsilon
$$

Now for $m, n \geq n_{0}$, we have

$$
\begin{aligned}
\rho\left(\frac{f_{n+m}-f_{n}}{2}\right) & \leq \frac{1}{2} \rho\left(f_{n+m}-c^{*}\right)+\frac{1}{2} \rho\left(f_{n}-c^{*}\right) \\
& \leq \rho\left(f_{n_{0}}-c^{*}\right) \\
& <\varepsilon .
\end{aligned}
$$

Hence $\left\{f_{n}\right\}$ is a $\rho$-Cauchy sequence in a $\rho$-closed subset $D$ of $L_{\rho}$, and so it must converge in $D$. Let $\lim _{n \rightarrow \infty} f_{n}=q$. That $q$ is a fixed point of $T$ now follows from Theorem 2 .

We now give some examples. The first one shows the existence of a mapping satisfying the condition (I) whereas the second one shows the existence of a mapping satisfying all the conditions of Theorem 3.

Example 1 Let $L_{\rho}=M[0,1]$ (the collection of all real valued measurable functions on $[0,1])$. Note that $M[0,1]$ is a modular function space with respect to

$$
\rho(f)=\int_{0}^{1}|f| .
$$

Let $D=\left\{f \in L_{\rho}: \frac{1}{2} \leq f(x) \leq 1\right\}$. Obviously $D$ is a nonempty closed and convex subset of $L_{\rho}$. Define $T: D \rightarrow C_{\rho}\left(L_{\rho}\right)$ as

$$
T f=\left\{g \in L_{\rho}: \frac{1}{2} \leq g(x) \leq 1+\frac{f(x)}{2}\right\} .
$$

Define a continuous and nondecreasing function $l:[0, \infty) \rightarrow[0, \infty)$ by $l(r)=\frac{r}{2}$. It is obvious that $\operatorname{dist}_{\rho}(f, T f) \geq l\left(\operatorname{dist}_{\rho}\left(f, F_{T}\right)\right)$ for all $f \in D$. Hence $T$ satisfies the condition (I).

Example 2 The real number system $\mathbb{R}$ is a space modulared by $\rho(f)=|f|$. Let $D=[1,2]$. Obviously $D$ is a nonempty closed and convex subset of $\mathbb{R}$. Define $T: D \rightarrow P_{\rho}(D)$ as

$$
T f=\left[1,1+\frac{f}{2}\right] \text {. }
$$

Define a continuous and nondecreasing function $l:[0, \infty) \rightarrow[0, \infty)$ by $l(r)=\frac{r}{4}$. It is obvious that $\operatorname{dist}_{\rho}(f, T f) \geq l\left(\operatorname{dist}_{\rho}\left(f, F_{T}\right)\right)$ for all $f \in D$. 
Note that $P_{\rho}^{T}(f)=\{f\}$ when $f \in D$. Hence $P_{\rho}^{T}$ is nonexpansive. Moreover, by Lemma 2, $P_{\rho}^{T}(f)=\{f\} \Longrightarrow f \in T f$ for all $f \in D$. Thus $\left\{f_{n}\right\} \subset D$ defined by $f_{n+1}=\left(1-\alpha_{n}\right) f_{n}+\alpha_{n} u_{n}$ where $u_{n} \in P_{\rho}^{T}\left(f_{n}\right) \rho$-converges to a fixed point of $T$.

\section{Competing interests}

The authors declare that they have no competing interests.

\section{Authors' contributions}

Both authors worked on the manuscript. Both read and approved the final manuscript.

\section{Author details}

'Department of Mathematics, Statistics and Physics, Qatar University, Doha, 2713, Qatar. ²Department of Mathematics and Applied Mathematics, University of Pretoria, Lynnwood road, Pretoria, 0002, South Africa.

\section{Acknowledgements}

The first author owes a lot to Professor Wataru Takahashi from whom he started learning the very alphabets of Fixed Point Theory during his doctorate at Tokyo Institute of Technology, Tokyo, Japan. He is extremely indebted to Professor Takahashi and wishes him a long healthy active life. The authors are thankful to the anonymous referees for giving valuable comments.

Received: 3 October 2013 Accepted: 24 January 2014 Published: 11 Feb 2014

\section{References}

1. Nakano, H: Modular Semi-Ordered Spaces. Maruzen, Tokyo (1950)

2. Musielak, J, Orlicz, W: On modular spaces. Stud. Math. 18, 591-597 (1959)

3. Khamsi, MA, Kozlowski, WM, Reich, S: Fixed point theory in modular function spaces. Nonlinear Anal. 14, 935-953 (1990)

4. Khamsi, MA: A convexity property in modular function spaces. Math. Jpn. 44, 269-279 (1996)

5. Dhompongsa, S, Benavides, TD, Kaewcharoen, A, Panyanak, B: Fixed point theorems for multivalued mappings in modular function spaces. Sci. Math. Jpn. e-2006, 139-147 (2006)

6. Benavides, TD, Khamsi, MA, Samadi, S: Asymptotically non-expansive mappings in modular function spaces. J. Math. Anal. Appl. 265, 249-263 (2002)

7. Benavides, TD, Khamsi, MA, Samadi, S: Asymptotically regular mappings in modular function spaces. Sci. Math. Jpn. 53, 295-304 (2001)

8. Benavides, TD, Khamsi, MA, Samadi, S: Uniformly Lipschitzian mappings in modular function spaces. Nonlinear Anal. $46,267-278(2001)$

9. Kumam, P: Fixed point theorem for non-expansive mappings in modular spaces. Arch. Math. 40, 345-353 (2004)

10. Kozlowski, WM: Modular Function Spaces. Dekker, New York (1988)

11. Dehaish, BAB, Kozlowski, WM: Fixed point iteration for asymptotic pointwise nonexpansive mappings in modular function spaces. Fixed Point Theory Appl. 2012, 118 (2012)

12. Markin, JT: Continuous dependence of fixed point sets. Proc. Am. Math. Soc. 38, 545-547 (1973)

13. Nadler, SB Jr.: Multivalued contraction mappings. Pac. J. Math. 30, 475-488 (1969)

14. Gorniewicz, L: Topological Fixed Point Theory of Multivalued Mappings. Kluwer Academic, Dordrecht (1999)

15. Lim, TC: A fixed point theorem for multivalued nonexpansive mappings in a uniformly convex Banach spaces. Bull. Am. Math. Soc. 80, 1123-1126 (1974)

16. Kutbi, MA, Latif, A: Fixed points of multivalued mappings in modular function spaces. Fixed Point Theory Appl. 2009 , Article ID 786357 (2009)

17. Kilmer, SJ, Kozlowski, WM, Lewicki, G: Sigma order continuity and best approximation in $L_{\rho}$-spaces. Comment. Math. Univ. Carol. 3, 2241-2250 (1991)

18. Schu, J: Weak and strong convergence to fixed points of asymptotically nonexpansive mappings. Bull. Aust. Math Soc. 43, 153-159 (1991)

19. Senter, HF, Dotson, WG: Approximating fixed points of nonexpansive mappings. Proc. Am. Math. Soc. 44(2), 375-380 (1974)

10.1186/1687-1812-2014-34

Cite this article as: Khan and Abbas: Approximating fixed points of multivalued $\rho$-nonexpansive mappings in modular function spaces. Fixed Point Theory and Applications 2014, 2014:34 